\title{
The Aquation of Po(IV): A Quantum Chemical Study.
}

Cite as: AIP Conference Proceedings 963, 909 (2007); https://doi.org/10.1063/1.2836239

Published Online: 08 January 2008

R. Ayala, J. M. Martinez, R. R. Pappalardo, A. Munoz-Paez, and E. Sanchez Marcos

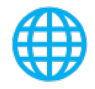

\section{Lock-in Amplifiers up to $600 \mathrm{MHz}$

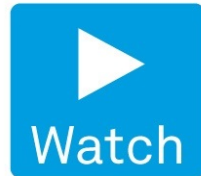

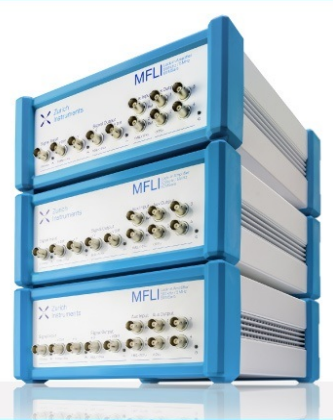




\title{
The Aquation of Po(IV): A Quantum Chemical Study.
}

\author{
R. Ayala*, J.M. Martinez ${ }^{\dagger}$, R.R. Pappalardo ${ }^{\dagger}$, A. Munoz-Paez ${ }^{*}$ and E. Sanchez \\ Marcos $^{\dagger}$ \\ *University of Seville. CSIC-ICMSE. Dept. of Inorganic Chemistry. \\ ${ }^{\dagger}$ University of Seville. Dept. of Physical Chemistry.
}

\begin{abstract}
The aim of this work is to present theoretical results of the hydration of the Po(IV) in solution. Particular attention is paid to the level of calculation needed to properly describe the system under study: $\mathrm{Po}$ (IV) coordination number in the first hydration shell and the nature of the polonium-water bonding. The hydration number of the Po(IV) is found to be in solution between 8 and 9 and the solvation free energy around $-1450 \mathrm{kcal} / \mathrm{mol}$. The $\mathrm{Po}-\mathrm{H}_{2} \mathrm{O}$ bonding is dominated by the strong electrostatic contribution although the peculiar geometry adopted by the different hydrates is due to covalent contributions. This involves the empty $6 \mathrm{p}$ orbital of the polonium ion and one lone pair on the oxygen atom of the water molecule. No role of the $6 \mathrm{~s}$ orbital of the polonium ion is detected.
\end{abstract}

Keywords: Aqueous solution; Ionic hydration; Quantum Chemistry, Polarizable Continuum Methods.

PACS: 61.20.Gy, 61.20.Qg, 61.20.Ja

\section{INTRODUCTION.}

Despite of the fact that one of the Marie Curie's pioneering research[1] was the discovery of the polonium metal extracted from pitchblende, the chemistry and properties of this element and its complexes are scarcely known. This fact is mainly due to two reasons. On one hand, polonium is a very rare element in Nature. It is found in uranium ores at about 100 micrograms per metric ton. On the other hand, polonium is highly toxic. The main hazard is its intense radioactivity (as an alpha and gamma emitter), which makes it very difficult to handle safely. Polonium has 25 known isotopes, all of which are radioactive. ${ }^{210} \mathrm{Po}$ is the most widely available, being its half-life 138.376 days. Even at very low concentrations (at traces level) the isotope ${ }^{210}$ Po leads to adverse health effects becoming lethal. In a case of individual and environmental contamination, the nature of chemical forms that are present are far from being understood. Therefore, elucidating and understanding polonium complexes can help us to develop protocols and strategies to minimize its harmful effects in living beings.

Although it is known that Polonium metal salts dissolves readily in dilute acids but is only slightly soluble in alkalis, its chemistry in solution has been scarcely studied. Fundamental aspects such as, its coordination numbers or hydration free energy in aqueous solutions are not known.

In this work, we have focused on the theoretical study of the ionic solutions of polonium, namely, the hydration of Po(IV) via polarizable continuum methods (PCM).[2] The absence of previous studies either experimental or theoretical on the hydration of the Po(IV) in water makes us to raise this study from the very beginning. Particular attention is devoted to the level of calculation needed to properly describe the system under study, the coordination number of the $\mathrm{Po}^{4+}$ in the first hydration shell and the nature of polonium-water bonding.

\section{METHODOLOGY}

Ab initio (HF, MP2 and CCSD(T)) and DFT (B3LYP, G96LYP and MPW1PW91) levels of computation were used to carry out optimizations of $\left[\mathrm{Po}\left(\mathrm{H}_{2} \mathrm{O}\right)_{n}\right]^{4+}$ clusters in gas phase and in solution. All the calculations were carried out with the Gaussian 03 program, except $\operatorname{CCSD}(\mathrm{T})$ calculations which were performed with the NWCHEM program. The Dunning double-zeta aug-cc-pVDZ were used as the basis sets of $\mathrm{O}$ and $\mathrm{H}$. For the Po, the relativistic small core pseudopotential (60 core electrons) developed by Dolg and co-workers were used for the core electrons while the valence electrons were described with the aug-cc-pVDZ basis set. The choice of Dunning basis sets was motivated by consistency with the limited availability of high quality small core pseudopotential and basis sets for the polonium atom. $\left[\mathrm{Po}\left(\mathrm{H}_{2} \mathrm{O}\right)_{n}\right]^{4+}(n=1-9)$ clusters were fully optimized firstly in gas phase. The former structures that were 
characterized as minimum structures (no negative frequencies) were taken as a starting point for the optimizations in solution via polarizable continuum (PCM )methods. Namely, Integral equation formalism polarizable continuum methods (IEFPCM) was used. $[3,4,5,6]$ A dielectric constant of 78.39 was used corresponding to that for liquid water at $25^{\circ} \mathrm{C}$. The geometries were fully reoptimized using this model to seek their structures in aqueous solution. Thus, bulk effects were taken into account on both energies and geometries. In the IEFPCM model, the solute is embedded in a cavity adapted to the molecular shape, which is defined by interlocking spheres centered on each atom or group. Cavity effects in the IEFPCM approach were studied using the following radii: $1.52 \AA$ for $\mathrm{O}, 1.20 \AA$ for $\mathrm{H}$ and 2.354 $\AA$ for Po. [7, 8] The radii have been multiplied by a standard factor 1.2 which accounts the fact that atomic bond or lone pair centers of the solvent molecules are normally located a bit further from the solute atoms than van der Waals radius.[2] Free energies of the clusters in gas phase and in solution were calculated. The latter were computed by combining thermodynamic free energies in the gas phase with a dielectric continuum model to treat solvent effects.

\section{RESULTS AND DISCUSSION.}

\section{Choice of computation level.}

In order to know the level of the computation that must be used to properly study $\left[\mathrm{Po}\left(\mathrm{H}_{2} \mathrm{O}\right)_{n}\right]^{4+}$ clusters, different ab initio (HF, MP2, CCSD(T)) and DFT (B3LYP, G96LYP, MPW1PW91) methods were tested. Differences among methods are basically in the Po-O distances and interaction energies. In the absence of experimental data, we assume that an accurate description of energetic and structural properties of the $\mathrm{Po}-\mathrm{H}_{2} \mathrm{O}$ clusters will be given by high level ab initio calculations such as $\operatorname{CCSD}(T)$. The excellent agreement between $\operatorname{CCSD}(\mathrm{T})$ and experimental estimations provides confidence that this level of theory will predict accurate properties such as bonding distances and interaction energies. However, while $\operatorname{CCSD}(\mathrm{T})$ calculations are of high accuracy, they are too computationally expensive and affordable in full optimizations of clusters with $n>4$. Nonetheless, results for small clusters optimized at $\operatorname{CCSD(T)}$ level can be used as a reference to evaluate the rest of the methods included in this study. As expected Po-O distances increases with the progress of the hydration for all the levels of computation. The interaction energy also increases with the number of hydrating water molecules but due to many body effects the energy per water molecule is reduced. This behaviour is in agreement with the elongation of the Po-O distance. The analysis of clusters with $n<4$ allows us the splitting of the methods considered in this study into two groups. On one hand, the methods whose results resemble to a certain extent $\operatorname{CCSD}(\mathrm{T})$ results, that is, MP2, MPW1PW91 and B3LYP. On the other hand, methods whose results diverge from CCSD(T) ones, that is, HF and G96LYP. Moreover, the larger $n$ is, the more similar the results of MP2 and MPW1PW91 are. Due to this fact and considering that MP2 and MPW1PW91 results are close to $\operatorname{CCSD}(\mathrm{T})$ results and MPW1PW91 is less cpu time demanding than MP2, we conclude that MPW1PW91 is the most suitable method to properly study $\left[\mathrm{Po}\left(\mathrm{H}_{2} \mathrm{O}\right)_{n}\right]^{4+}$ clusters at affordable cost. Of course, this conclusion is a result of a compromise between accuracy and feasibility.

\section{Semi-continuum solvation model.}

Once the level of computation has been chosen the following step is related with the computation of free energies in solution $\left(\Delta \mathrm{G}_{\text {solv }}\right)$ at MPW1PW91 level.

The continuum-discrete model used in this study takes into account the cavity containing the solute and its first hydration shell and solvent medium surrounding the cavity defined solely by its dielectric constant. Induced charges on the surface of the cavity due to solvent-solute polarization allow one to obtain the free energy of the Po(IV) in solution. As shown in a number of previous studies of monoatomic cations the inclusion of explicit solvent molecules in the first hydration shell is necessary to converge to reliable values of the free energy of solvation. $[9,10,11]$ Inclusion of the second or outer order solvation shells is generally not used since their effect is less important and the difficulties to evaluate the statistical averaging when the number of water molecules increases.[10]

Solvation energies are collected in Table 1. Examination of the data reveals that the solution energy contribution depends on cluster size. The non-electrostatic contribution $\left(\Delta \mathrm{G}_{c a v}+\Delta \mathrm{G}_{d i s p-r e p}\right)$ are rather independent on the cluster considered whereas the formation of the cluster and the electrostatic component $\left(\Delta \mathrm{G}_{\text {clus }}+\Delta \mathrm{G}_{\text {cont }}\right)$ are responsible for the main differences. The lack of experimental data prevents us a direct validation of our results. However, the comparison of our results with experimental data of other tetravalent cations such as $\mathrm{Zr}^{4+}, \mathrm{Sn}^{4+}, \mathrm{Ce}^{4+}, \mathrm{Hf}^{4+}, \mathrm{Th}^{4+}$, 
TABLE 1. Solvation free energies $\left(\Delta \mathrm{G}_{\text {solv }}\right)$ in $\mathrm{kcal} / \mathrm{mol} . \Delta \mathrm{G}_{\text {solv }}=$ $\Delta \mathrm{G}_{\text {cluster }}+n \Delta \mathrm{G}_{\text {vap }}+\Delta \mathrm{G}_{\text {cov }}+\Delta \mathrm{G}_{\text {dis-rep }}+\Delta \mathrm{G}_{\text {cont }}$.

\begin{tabular}{|c|c|c|c|c|c|}
\hline$n$ & $\Delta \mathrm{G}_{\text {cluster }}$ & $\Delta \mathrm{G}_{\text {vap }}$ & $\Delta \mathrm{G}_{\text {cav }+ \text { rep }+ \text { dis }}$ & $\Delta \mathrm{G}_{\text {cont }}$ & $\Delta \mathrm{G}_{\text {solv }}$ \\
\hline 6 & -762.9 & 22.2 & 16.2 & -696.7 & -1421.2 \\
7 & -800.8 & 25.9 & 16.4 & -680.1 & -1438.6 \\
8 & -831.9 & 29.6 & 17.4 & -659.0 & -1443.9 \\
9 & -851.5 & 33.3 & 17.7 & -644.4 & -1444.9 \\
\hline
\end{tabular}

$\mathrm{Pa}^{4+}, \mathrm{U}^{4+} . \mathrm{Np}^{4+}$ and $\mathrm{Pu}^{4+}$ indicates that the estimations obtained in this study predict solvation free energies that are contained in the interval $([1380-1800] \mathrm{kcal} / \mathrm{mol})$ defined by these cations. Moreover, the Po-O distances [2.1-2.5 A] are also in agreement with the experimental results of tetravalent cations in aqueous solutions.[12]

The $\left[\mathrm{Po}\left(\mathrm{H}_{2} \mathrm{O}\right)_{9}\right]^{4+}$ cluster seems to be the most stable cluster in solution. However, the difference in energy with the $\left[\mathrm{Po}\left(\mathrm{H}_{2} \mathrm{O}\right)_{8}\right]^{4+}$ cluster is negligible, specially taking into account that free energy of the cluster formation was computed from gas phase results. We can conclude that on the basis of our study, the number of water molecules in the first hydration shell of the Po(IV) is between 8 and 9 .

\section{Nature of $\mathrm{Po}_{\mathrm{H}} \mathrm{H}_{2} \mathrm{O}$ bonding.}

In order to properly describe ion hydration, not only it is important to have a good command of coordination numbers and solvation free energies but also a detailed understanding of the nature of the ion-solvent bonding. To study the driving force of the $\mathrm{Po}-\mathrm{H}_{2} \mathrm{O}$ interaction a Natural Bond Order (NBO) analysis was performed. The analysis of the $\left[\mathrm{Po}\left(\mathrm{H}_{2} \mathrm{O}\right)_{n}\right]^{4+}$ clusters with $n=1-7$ shows that there is bond between the $6 p$ orbitals of polonium ion and the water molecules. Nonetheless, the $6 s$ orbital of the polonium ion is not involved in the bonding. For $n=8$ and 9 , the NBO analysis indicates that there is also participation of the $6 d$ orbitals of the polonium ion in the bonding but not of the $6 s$ orbital. The latter can be considered a core orbital. This way, the $\mathrm{OPoO}$ angle in $\left[\mathrm{Po}\left(\mathrm{H}_{2} \mathrm{O}\right)_{n}\right]^{4+}(n=2-6)$ is about 90 or 180 degrees as a result of the interaction of the water molecules with the $6 p$ orbitals of the polonium ion (Fig.1). The lack of hybridization between the $6 p$ and $6 s$ polonium orbital precludes the formation of small clusters where the water molecules are simmetrycally distributed around the ion minimizing water-water repulsion and maximizing ion-water attraction. Although orbital interaction is responsible for the structure of the clusters, the electrostatic and its associated long range contributions retain most of the $\mathrm{Po}-\mathrm{H}_{2} \mathrm{O}$ interaction, especially in the case of larger clusters.

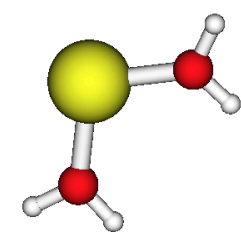

$n=2$

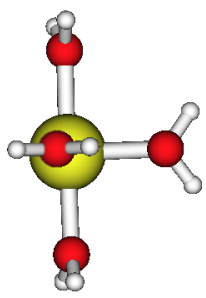

$n=4$
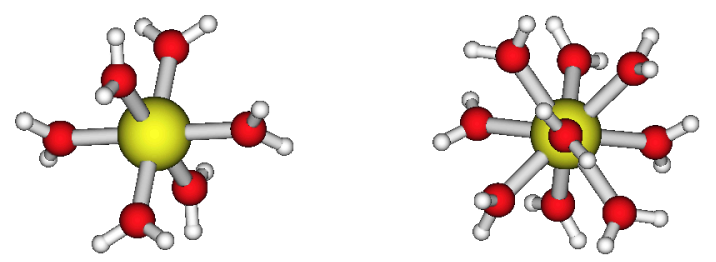

$n=9$

FIGURE 1. Selected $\left[\mathrm{Po}\left(\mathrm{H}_{2} \mathrm{O}\right)_{n}\right]^{4+}$ elusters optimized at MPW1PW91 level.

\section{CONCLUDING REMARKS.}

In this work we present a computational study of the $\mathrm{Po}(\mathrm{IV})$ hydration. $\left[\mathrm{Po}\left(\mathrm{H}_{2} \mathrm{O}\right)_{n}\right]^{4+}$ clusters were optimized in gas phase and in solution. MPW1PW91 functional was proved to be the most suitable level of calculation to properly describe $\mathrm{Po}-\mathrm{H}_{2} \mathrm{O}$ interactions at affordable cost. Solvent effects were taken into account via the PCM model. We conclude that the hydration number of the Po(IV) is ruled by a dynamic equilibrium involving the octa- and ennea- 
hydrates, and the solvation free energy is around $-1450 \mathrm{kcal} / \mathrm{mol}$. The $\mathrm{Po}-\mathrm{H}_{2} \mathrm{O}$ bonding was found to be mainly between the $6 p$ orbital of the polonium ion and the water molecules with no role of the $6 s$ orbital of the polonium ion.

\section{ACKNOWLEDGMENTS}

Spanish DGICYT (CTQ2005-03657) and Junta de Andalucia (group FQM282) are acknowledged for financial support.

\section{REFERENCES}

1. P. Curie, and M. P. Curie, Comptes Rendus 127, 175 (1898).

2. J. Tomasi, and M. Persico, Chem. Rev. 94, 2027 (1994).

3. M. Cances, B. Mennucci, and J. Tomasi, J. Chem. Phys. 107, 3032 (1997).

4. B. Mennucci, and J. Tomasi, J. Chem. Phys. 106, 5151 (1997).

5. B. Mennucci, E. Cances, and J. Tomasi, J. Phys. Chem. B 101, 10506 (1997).

6. J. Tomasi, B. Mennucci, and E. Cances, J. Mol. Struct. (Theochem) 464, 211 (1999).

7. C. Amovilli, and B. Mennucci, J. Phys. Chem. B 101, 1051 (1997).

8. B. Mennucci, and J. M. Martinez, J. Phys. Chem. B 109, 9818 (2005).

9. R. R. Pappalardo, E. Sanchez Marcos, and D. Rinaldi, J. Phys. Chem. 95, 8928 (1991).

10. J. M. Martinez, R. Pappalardo, and E. Sanchez Marcos, J. Phys. Chem. A 101, 4444 (1997).

11. C.-G. Zhan, and D. Dixon, J. Phys. Chem. A 108, 2020 (2004).

12. H. Ohtaki, and T. Radnai, Chem. Rev. 93, 1157 (1993). 